\title{
DOS NUEVAS ESPECIES DE PSEUDOSINELLA SCHÄFFER, 1897 (COLLEMBOLA, ENTOMOBRYIDAE) DE CASTILLA-LA MANCHA
}

\author{
J. C. Simón Benito ${ }^{1}$ \& J. Romera Becerro
}

\begin{abstract}
RESUMEN
Se han encontrado dos nueva especies de Pseudosinella (Collembola, Entomobryidae), $P$. lafargensis sp. n. y $P$. cementensis sp. n., en Castilla-La Mancha, en donde el número de citas de este género es muy reducido.

Palabras clave: Collembola, Pseudosinella, Castilla-La Mancha, España.
\end{abstract}

\section{ABSTRACT \\ Two new species of Pseudosinella Schäffer, 1897 (Collembola, Entomobryidae) from Castilla-La Mancha}

Two new species of Pseudosinella (Collembola, Entomobryidae), P. lafargensis sp. n. and $P$. cementensis sp. n., have been found in Castilla-La Mancha, where the number of citations of this genus is very few.

Key Words: Collembola, Pseudosinella, Castilla-La Mancha, Spain.

\section{Introducción}

En algunas regiones de la Península Ibérica el genero Pseudosinella ha sido muy bien estudiado, cornisa Cantábrica, Pirineos, Levante..., mientras que en otras se carece prácticamente de información, una de estas últimas es Castilla-La Mancha. De un total de 60 especies de este género sólo 3 especies se han localizado en dicha comunidad: Pseudosinella arretzi Simón, 1979, P. selgae Simón, 1978 y P. suboculata Bonet, 1931, las dos primeras en la provincia de Toledo (Simón, 1978; Luciáñez \& Simón, 1993) y la ultima en Cuenca y Guadalajara (Gama, 1985). Este trabajo extiende el conocimiento del citado género a la provincia de Toledo, ya que se se ha estudiado la recolonización de la fauna de una cantera en Yepes-Ciruelos, originariamente suelo agrícola que se está transformando en un medio con vegetación seminatural, encontrándose hasta ahora dos nuevas especies para la ciencia: Pseudosinella lafargensis sp. n. y Pseudosinella cementensis sp. n.

\footnotetext{
Comisión Docente de Zoología, Departamento de Biología, C/ Darwin, 2, Universidad Autónoma de Madrid, 28049 Madrid, Spain, e-mail: carlos.simón@uam.es
} 


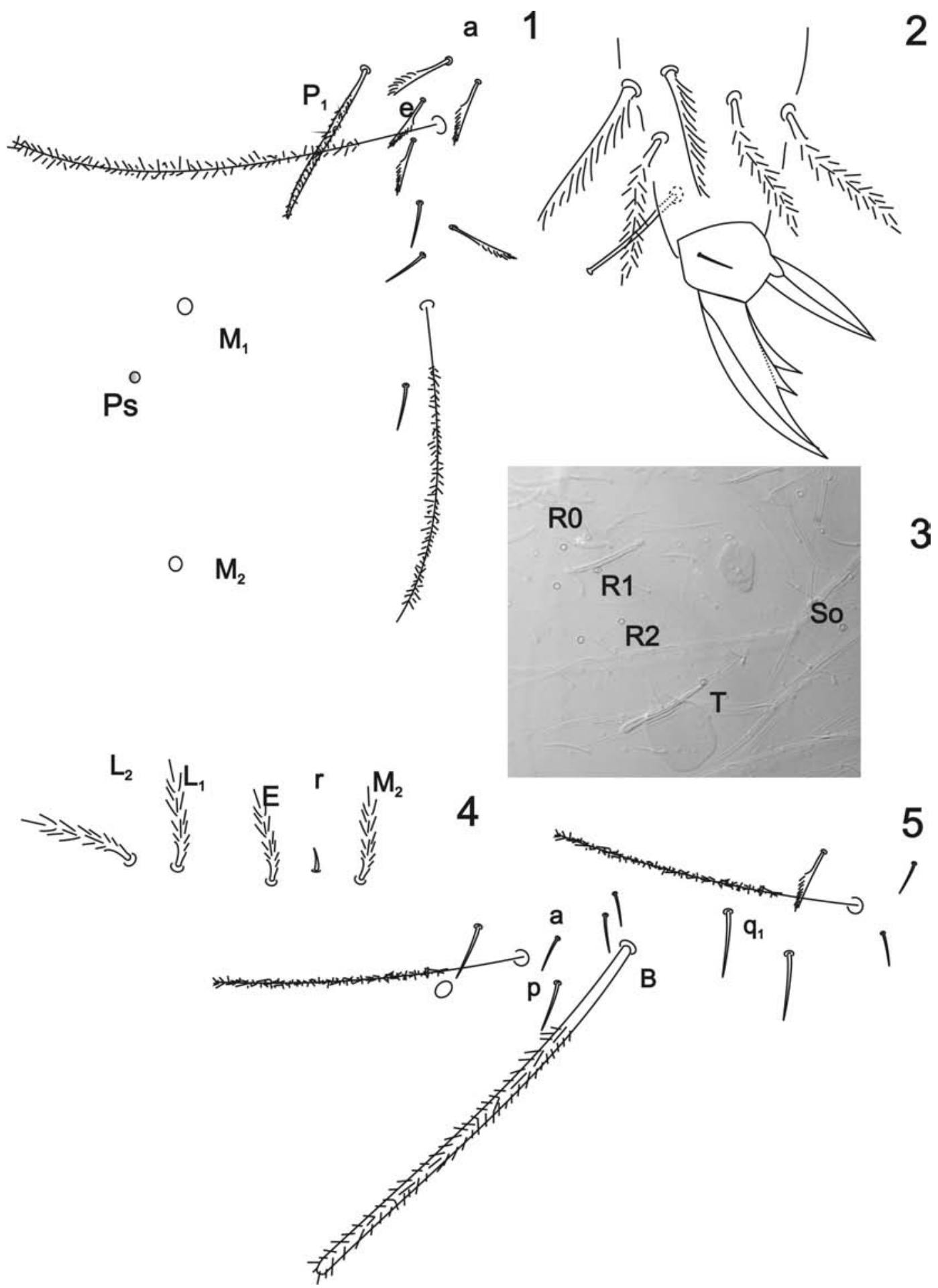

Figs.1-5.- Pseudosinella lafargensis sp. n.: 1) Quetotaxia del II segmento abdominal; 2) Quetotaxia del IV segmento abdominal, $\mathrm{P}=$ macroseda anterior, $\mathrm{M}=$ macroseda mediana, $\mathrm{Ps}=$ Pseudoporo; 3) Uña del III par de patas; 4) Labio; 5) Quetotaxia cefálica.

Figs. 1-5.- Pseudosinella lafargensis sp. n.: 1) Chaetotaxy of abdominal II; 2) Chaetotaxy of anterior bothriotrichal complex of Abdominal IV, $\mathrm{P}_{1}=$ previous macrochaeta ; $\mathrm{M}=$ median macrochaeta, $\mathrm{Ps}=$ Pseudopore; 3 ) Tibiotarsus III, unguis and unguiculus; 4) Labium; 5) Chaetotaxy of the head. 


\section{Pseudosinella lafargensis sp. $\mathbf{n}$.}

Material TIPO: Toledo, Yepes-Ciruelos, 15 de Mayo de 2007. Uso del suelo: cantera, vegetación ruderal, 1 ejemplar en la muestra $25 \mathrm{~S}$ y tres ejemplares en la muestra $17 \mathrm{~S}$, uno de ellos el holotipo macho.

Holotipo y los tres paratipos depositados en el Laboratorio de Entomología del Departamento de Biología, de la Universidad Autónoma de Madrid.

DESCRIPCIÓN. Longitud máxima: $0,75 \mathrm{~mm}$. Sin pigmento en el cuerpo, sin ojos. Antenas sin pigmento, su relación con la diagonal de la cabeza es de 1,4. La relación de los artejos antenales I:II:III:IV es 10:20:15:32. Antenal IV sin vesícula apical redondeada. Órgano sensorial del artejo antenal III formado por dos bastones ligeramente inclinados.

La fórmula del labio es: -, $\mathrm{M}_{2}, \mathrm{r}, \mathrm{E}, \mathrm{L}_{1}, \mathrm{~L}_{2} . \mathrm{M}_{1}$ ausente, $r$ es una microseda lisa (Fig. 4). Papila labial externa con una seda recta que no llega al ápice de la misma. Con tres sedas en el lóbulo externo de la maxila. Tres sedas ciliadas a lo largo del surco cefálico ventral. Con cinco papilas labrales triangulares.

La fórmula de las macrosedas dorsales es: R011/10/0101+2. Cabeza con macrosedas R0, R1 y R2, T y So, macrosedas, R3 y S ausentes (Fig. 5). Quetotaxia del II segmento abdominal: $\mathrm{paBq}_{1} \mathrm{q}_{2}$, (Fig. 1). Seda accesoria "s" junto al botriotrico anterior del IV segmento abdominal ausente, una de las macrosedas mediales está situada en una posición anterior al pseudoporo, la otra es posterior al mismo (Fig. 2).

Tibiotarso con un espolón mazudo, su relación con la longitud interna de la uña es de 0,80 . La uña sin diente impar, con un par de dientes basales, el más proximal situado en el $40 \%$ y el más distal en el $60 \%$ de la longitud de la cresta interna de la uña. Empodio puntiagudo liso, la relación con la cresta interna de la uña es de 0,70 (Fig. 3). Patas sin escamas.

Retináculo con $4+4$ dientes, sin sedas en la base. Mucrón bidentado y con una seda en su base. Placa manubrial con dos macrosedas internas y una externa.

ETIMOLOGÍA. El nombre de la especie alude al de la Empresa: Lafarge Asland, S.A. que ha financiado este trabajo.

COMENTARIos. En la Tabla 1 se dan las diferencias de la nueva especie con todas las que presentan la misma macroquetotaxia dorsal.

\section{Pseudosinella cementensis sp. $\mathbf{n}$.}

Material tiPo: Toledo, Yepes-Ciruelos, 15 de Mayo de 2007. Uso del suelo: cantera, vegetación ruderal, 1 ejemplar, el holotipo, en la muestra $1 \mathrm{~S}, 1$ ejemplar en la muestra $15 \mathrm{~S}$ y 1 ejemplar en la muestra $18 \mathrm{~S}$.

Tabla 1.- Caracteres diferenciadores entre las especies con la misma macroquetotaxia dorsal R011/10/0101+2.

Table 1.- Differential characters between species with the same dorsal macrochaetotaxy R011/10/0101+2.

\begin{tabular}{|c|c|c|c|c|c|c|}
\hline Especies & Ojos & $\begin{array}{c}\text { Labio } \\
\text { fórmula }\end{array}$ & II Ab & s IV & $\begin{array}{l}\text { Grueso diente } \\
\text { en el empodio }\end{array}$ & $\begin{array}{l}\text { Espolón } \\
\text { mazudo }\end{array}$ \\
\hline variabilis Gama \& Busmachiu, 2004 & 5 & MRELL & paBqq & - & - & + \\
\hline wahlgreni Börner, 1901 sensu Stomp, 1971 & 5 & MMrELL & paBqq & - & - & + \\
\hline zygophora Schille, 1908 sensu Stomp, 1971 & 5 & MMrELL & $\mathrm{paBq}$ & - & - & + \\
\hline cordobensis Simón, Bach \& Gajú, 1986 & 3 & MMrELL & $-\mathrm{aBqq}$ & + & - & + \\
\hline espagnoli Simón \& Selga, 1977 & 3 & MMrELL & $-\mathrm{aBqq}$ & - & + & + \\
\hline paprivata Ellis, 1976 & $1,2,3$ & M-ELL & $-\mathrm{Bqq}$ & - & - & + \\
\hline paprivata Ellis, 1976 sensu Gruia, Poliakov \& Broza, 1999 & 3 & M-ELL & $-\mathrm{aBqq}$ & - & - & + \\
\hline tietarensis Jordana \& Baquero, 2007 & 3 & MMrELL & $-\mathrm{aBqq}$ & + & - & + \\
\hline annemariae Stomp, 1972 & 3 & Mmrell & paBqq & + & - & + \\
\hline arretzi Simón, 1979 & 3 & MMrELL & $-a B q q$ & + & + & + \\
\hline aidamar Luciáñez \& Simón, 1994 & 2 & MMrELL & $-\mathrm{aBqq}$ & + & - & + \\
\hline angelae Gama, 2004 & 2 & MMrELL & paBqq & - & - & - \\
\hline cobosae Luciáñez \& Simón, 1994 & 2 & MMrELL & $-a B q q$ & + & + & + \\
\hline leclerci Stomp, Thibaud \& Massoud, 1982 & 2 & mmrell & pABqq* & + & - & - \\
\hline jordanai Simón \& Palacios-Vargas, 2007 & 1 & MMrELL & $-a B q q$ & - & - & - \\
\hline lafargensis sp.n. & 0 & -MrELL & paBqq & - & - & + \\
\hline
\end{tabular}

*esta especie la nominan con dos sedas, y la dibujan con una. 


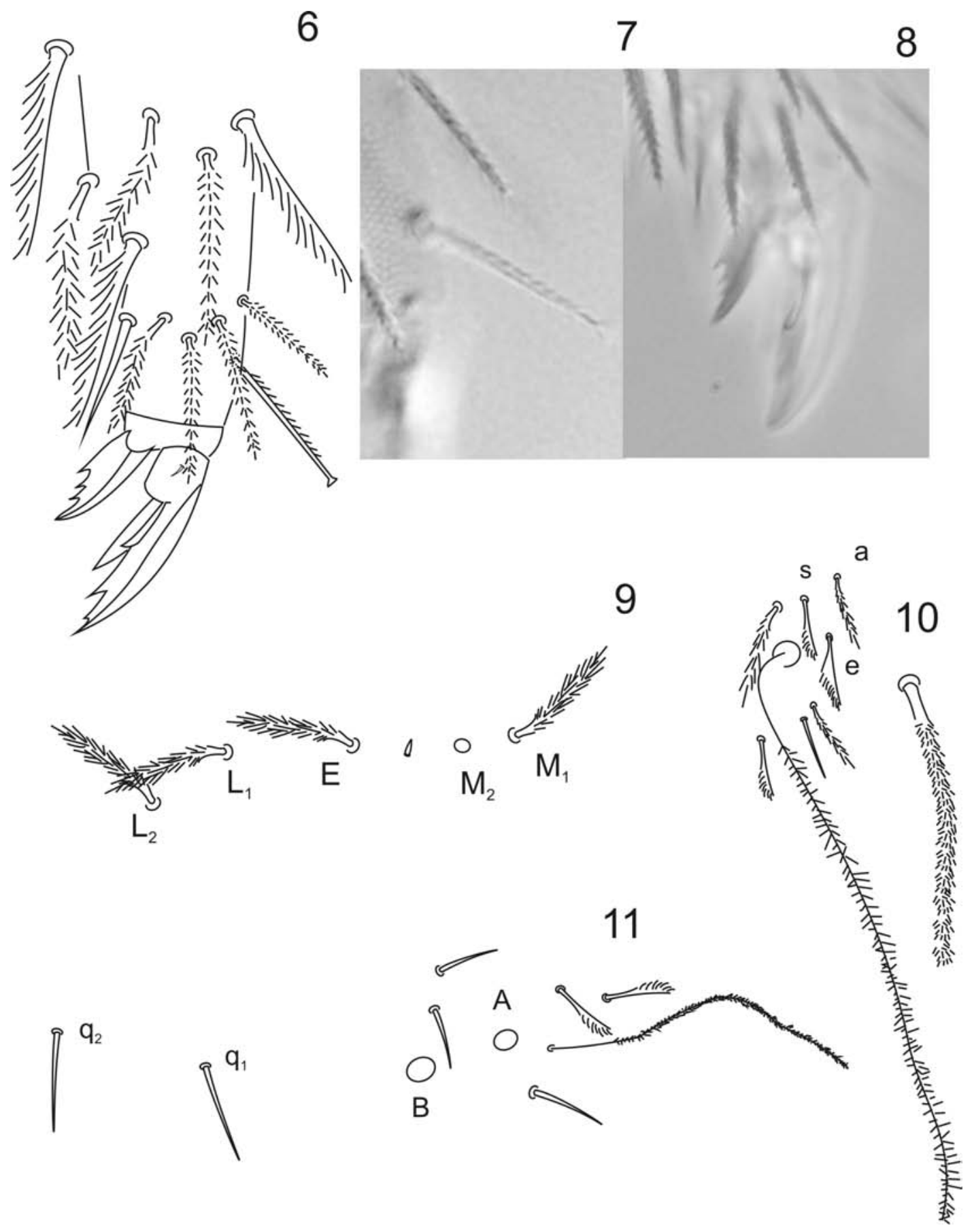

Figs. 6-11.—Pseudosinella cementensis sp. n.: 6) Tibiotarso, espolón y uña del III par de patas;7) Detalle del espolón; 8) Detalle de los dientes del emporio; 9) Labio; 10) Quetotaxia del IV segmento abdominal; 11) Quetotaxia del II segmento abdominal.

Figs. 6-11.- Pseudosinella cementensis sp. n.: 6) Tibiotarsus III, tenner hair, unguis and unguiculus;7) Tenner hair details; 8) Details of teeth empodium; 9) Labium; 10) Chaetotaxy of anterior bothriotrichal complex of Abdominal IV; 11) Chaetotaxy of abdominal II. 
Tabla 2.- Caracteres diferenciadores entre las especies con la misma macroquetotaxia dorsal R011/20/0201+2.

Table 2.- Differential characters between species with the same dorsal macrochaetotaxy R011/20/0201+2.

\begin{tabular}{|c|c|c|c|c|c|c|c|}
\hline Especies & $\begin{array}{c}\text { Labio } \\
\text { fórmula }\end{array}$ & R labio liso & II $\mathbf{A b}$ & $\begin{array}{c}\text { Dientes } \\
\text { internos uña }\end{array}$ & $\begin{array}{l}\text { Espolón } \\
\text { ciliado }\end{array}$ & $\begin{array}{l}\text { Empodio, } \\
\text { dientes }\end{array}$ & $\begin{array}{c}\text { Empodio, } \\
\text { forma }\end{array}$ \\
\hline decepta Gisin \& Gama, 1969 & MMRELL & - & $-\mathrm{AB} q \mathrm{q}$ & 1 & - & liso & puntiagudo \\
\hline pseudodecepta Arbea \& Jordana, 1990 & MMrELL & + & pABqq & 1 & - & aserrado & puntiagudo \\
\hline soriensis Simon \& Signoret, 2006 & MMrELL & + & $\mathrm{pABqq}$ & 1 & - & con 1 gran diente & truncado \\
\hline cementensis $\mathbf{s p .} \mathbf{n}$. & MMrELL & + & $\mathrm{pABqq}$ & 2 & + & con 3-4 dientes claros & truncado \\
\hline
\end{tabular}

Holotipo y los dos paratipos depositados en el Laboratorio de Entomología del Departamento de Biología, de la Universidad Autónoma de Madrid.

DESCRIPCIÓN. Longitud máxima: $0,95 \mathrm{~mm}$. Sin pigmento en el cuerpo. Antenas sin pigmento, su relación con la diagonal de la cabeza es de 1,6. La relación de los artejos antenales I:II:III:IV es 10:15:15:32. Antenal IV sin vesícula apical. Órgano sensorial del III artejo antenal formado por dos bastones rectos.

La fórmula del labio es: $\mathrm{M}_{1}, \mathrm{M}_{2}, \mathrm{r}, \mathrm{E}, \mathrm{L}_{1}, \mathrm{~L}_{2}$. $\mathrm{r}$ es una microseda lisa (Fig. 9). Papila labial externa con una seda recta que no llega al ápice de la misma. Con tres sedas en el lóbulo externo de la maxila. Tres sedas ciliadas a lo largo del surco cefálico ventral. Con cinco papilas labrales triangulares.

La fórmula de las macrosedas dorsales es: R011/20/0201+2. Cabeza con macrosedas R0, R1 y R2, T y So, macrosedas, R3 y S ausentes (Fig. 5). Quetotaxia del II segmento abdominal: pABq $q_{2}$, (Fig. 11). Seda accesoria "s" junto al botriotrico anterior del IV segmento abdominal presente (Fig. 10).

Tibiotarso con un espolón mazudo, con ciliación clara (Figs. 6, 7), la relación del mismo con la longitud de la cresta interna de la uña es de 0,95 . Uña con dos dientes medianos internos, el más alejado de la base se encuentra en el $87 \%$ de la misma, el otro, de mayor tamaño, se localiza en el $79 \%$ de ella, con un par de dientes basales, el proximal alcanza el $47 \%$ de la longitud de la cresta interna de la uña y el distal el 63\%. Empodio truncado con 34 dientecillos muy definidos en su borde externo (Figs. 6, 8), su relación con la cresta interna de la uña es de 0,60 (Fig. 6).

Retináculo con $4+4$ dientes, sin sedas en la base. Mucrón bidentado y con una seda en su base. Placa manubrial con dos macrosedas internas $\mathrm{y}$ una externa.
ETIMOLOGíA. El nombre de la especie alude al material que fabrica la empresa Lafarge Asland, S.A. que ha financiado este trabajo.

Comentarios. En la Tabla 2 se dan las diferencias de la nueva especie con todas las que presentan la misma macroquetotaxia dorsal.

\section{AGRADECIMIENTOS}

Este trabajo ha sido financiado mediante un contrato $\mathrm{I}+\mathrm{D}$ entre Lafarge Asland, S.A. y la Universidad de Castilla-La Mancha denominado "Seguimiento de la restauración de la cantera de Lafarge Asland en Yepes-Ciruelos"

También queremos agraceder al Dr. R. Jordana la inestimable ayuda en la revisión de este manuscrito, y en las sugerencias para el esclarecimiento de las especies aquí descritas.

\section{Referencias}

ArbeA, J. L. \& JordanA, R., 1989. New species of Pseudosinella and Lepidocyrtus from Navarra (Northern Iberian Peninsula). Spixiana, 13: 25-31.

GamA, M. M., 1985. Collemboles cavernicoles de L'Espagne II (Insecta, Apterygota). Miscelanea Zoologica, 9: 209-214.

GAma, M. M., 2004. Une espèce nouvelle de Pseudosinella provenant du Portugal (Insecta: Collembola). XVIIe contribution. Revue suisse Zoologie, 111: 563565.

Gama, M. M. \& Busmachiu, G., 2004. New species of the genus Pseudosinella fromMoldavia and Ukranie (Collembola: Entomobryidae). XVIII contributrion. Revue Suisse de Zoologia, 111: 563-565.

JordanA, R. \& BAQUERO, E., 2007. New species of Pseudosinella Schäffer, 1897 (Collembola, Entomobryidae) from Spain. Zootaxa, 1465: 1-14.

LuCiáñEz, M. J. \& Simón, J. C., 1993. Estudio de las poblaciones de colémbolos en suelos cultivados de la 
provincia de Toledo. Actas XII Congreso LatinoAmericano de Ciencias del Suelo; Salamanca: 464471.

LuCiáñez, M. J. \& Simón, J. C., 1994. Cinco especies nuevas del genero Pseudosinella (Collembola: Entomobryidae) de la Península Ibérica. Annales de la Société Entomologique de France, 30: 319-327.

Simón, J. C., 1978. Descripción de Pseudosinella selgae nov. sp. y nuevas citas de P. templadoi procedentes de suelos de la Península Ibérica. Graellsia, 52: 235-241.
Simón, J. C. \& Palacios-Vargas, J. G., 2007. New species of Pseudosinella (Collembola: Entomobryidae) from Iberian Peninsula. Zootaxa, 1479: 9-19.

Recibido, 10-XII-2007

Aceptado, 13-V-2007

Publicado, 30-VI-2007 